\title{
Quality of Drug Treatment of Childhood Persistent Asthma in Maryland Medicaid Recipients in Transition From Managed Fee for Service to Managed Capitation
}

\author{
Puneet K Singhal, BPharm, PhD; llene Zuckerman, PharmD, PhD; \\ Bruce Stuart, PhD; Laurence Magder, PhD; and Haya Rubin, MD, PhD
}

\begin{abstract}
BACKGROUND: From December 1991 to June 1997, approximately $80 \%$ of Maryland's Medicaid recipients were served through a fee-for-service (FFS) managed care delivery system in which assigned primary care providers served as gatekeepers for hospital and specialty services. The remaining $20 \%$ of recipients were voluntarily enrolled in 1 of 5 available health maintenance organizations (HMOs). Beginning in June 1997, Maryland required most Medicaid recipients to enroll in capitated managed care organizations (MCOs), also referred to as managed Medicaid plans. Although research has been conducted on the quality of asthma care among MCOs and in MCOs for Medicaid versus non-Medicaid members, the quality of asthma care has been less well studied for MCO patients than for FFS patients.
\end{abstract}

OBJECTIVE: To determine whether quality of drug use among Medicaid children with persistent asthma was different after the transition from the managed care FFS system to a capitated managed Medicaid system.

METHODS: This 4-year retrospective cohort study (from June 1, 1996, to December 31, 2000) followed children aged 5 to 18 years with persistent asthma (defined by the existence of at least 1 medical claim with an International Classification of Diseases, Ninth Revision, Clinical Modification diagnosis code of 493.x and receipt of 2 or more pharmacy claims for

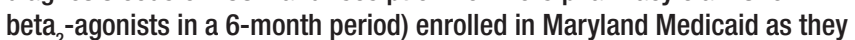
transitioned from the managed FFS system to 1 of 4 large capitated MCOs. Children were selected from a review of Medicaid enrollment records and medical and pharmacy FFS claims filed between June 1, 1996, and December 31,1997 . Children with a diagnosis of cystic fibrosis were excluded. The asthma quality indicator was defined as the proportion of children with persistent asthma (who had 2 or more claims for any short-acting beta ${ }_{2}-$ agonists [SABAs], including metered-dose inhalers, nebulizers, or oral forms, which we defined as rescue medication, within a 6-month period), who also had at least 1 claim for a controller medication (inhaled corticosteroid, mastcell stabilizer, or leukotriene-receptor modifier) in the same 6-month period. Subjects were followed from June 1, 1996 (or, if later, the first Medicaid eligibility date), through December 31, 2000 (or, if earlier, the last Medicaid eligibility date). Mean quality indicator rates were calculated for the 2 managed FFS periods (FFS1 and FFS2) and the 6 managed Medicaid 6-month periods. We used generalized estimating equations to test for significant trends over time and to compare changes in the quality indicator in the managed Medicaid plans.

RESULTS: There were 3,721 children who met the inclusion and exclusion criteria for the study. The quality indicator (proportion of patients who received a controller medication among those receiving SABAs for asthma) was $62 \%$ in managed FFS1 and $57 \%$ in managed FFS2. In the first 6 months of managed Medicaid plans, the quality indicator rose from $56 \%$ to $57 \%$, $59 \%, 61 \%, 66 \%$, and $59 \%$ in the ensuing five 6 -month observation periods. The results from the generalized estimating equations suggested slight improvement in the quality indicator in the managed Medicaid plans, but the difference was not significant (relative risk 1.01, 95\% confidence interval, 0.95-1.08). There was no significant trend in the asthma quality indicator over time in the managed Medicaid plans.
CONCLUSION: There was no distinct improvement or worsening in asthma care as measured by the quality indicator (proportion of patients who received a controller medication among those receiving SABAs for asthma) as children moved from managed FFS to managed Medicaid. Larger sample sizes with no data loss may have produced a different result.

KEYWORDS: Medicaid managed care, Managed Medicaid, Fee-for-service, Quality indicator, Asthma, Children

J Manag Care Pharm. 2007;13(4):310-18

Copyright@ 2007, Academy of Managed Care Pharmacy. All rights reserved.

\section{What is already known about this subject}

- Studies evaluating access and quality of care in managed Medicaid versus FFS Medicaid plans have reported results that are mixed but that generally favor managed care.

- Almost all of the published work makes cross-sectional comparisons between care received in managed Medicaid and FFS Medicaid, and no study has assessed care quality in a longitudinal analysis.

\section{What this study adds}

- This longitudinal cohort design without a control group found that quality of care, defined as the proportion of children with persistent asthma who received a controller medication as well as SABAs, was no different in two 6-month managed Medicaid FFS periods than in six 6-month managed Medicaid periods

- A quality indicator calculated as the ratio of Medicaid children in a 6-month period who have at least 2 pharmacy claims for acute rescue medications (SABAs) (the denominator) and at least 1 pharmacy claim for a controller medication (the numerator) requires large sample sizes to detect clinically meaningful quality improvement when measured longitudinally because of the absence of pharmacy claims for the cohort of patients in 1 or more 6-month periods.

\footnotetext{
Cince the 1990s, managed care has become the dominant form of health care delivery for Medicaid recipients in states seeking to curb health care costs and improve access and quality of care. According to recent statistics, about $63 \%$ of
} 


\section{Quality of Drug Treatment of Childhood Persistent Asthma in Maryland Medicaid \\ Recipients in Transition From Managed Fee for Service to Managed Capitation}

Medicaid recipients (28.6 million) in 49 states were enrolled in a Medicaid managed care organization (MCO, managed Medicaid plan) as of June 2005. ${ }^{1}$

Numerous studies have evaluated access and quality after transition from fee-for-service (FFS) to a managed Medicaid plan. ${ }^{2-}$ ${ }^{4}$ A 1993 General Accounting Office (GAO) report summarized the studies examining access and quality issues under several state managed Medicaid plans, focusing on a variety of quality indicators such as pregnancy outcomes and prenatal care, wellchild care and sick-child visits, preventive care for children and adults, and childhood immunization rates. ${ }^{2}$ The GAO report concluded that there was a slight improvement in access and the same level of quality of care in managed Medicaid plans as in the traditional FFS plan.

Another study reviewed access and quality in 6 Medicaid demonstration projects. $^{3}$ The study found that access as perceived in Medicaid consumer surveys was generally greater for the demonstration (managed Medicaid) sites than for nondemonstration (FFS) comparison sites. However, access measured using objective measures, such as travel time and waiting time for appointments and in physicians' offices, was mixed between sites. Further, the review found that quality of care, assessed by using chart abstractions, showed no differences for birth outcomes and complications between demonstration and comparison sites. The other acute and chronic conditions studied for females, such as vaginitis, urinary tract infection, pelvic inflammatory disease, and hypertension, presented a mixed picture: equivalent or slightly worse care in 1 demonstration (managed Medicaid) site and equivalent or somewhat better care in another demonstration (managed Medicaid) site compared with FFS comparators.

Another review summarized access and quality of care studies for Arizona's managed Medicaid plan and New Mexico's FFS plan. ${ }^{4}$ The review found that children and pregnant women received earlier, more frequent, and more complete health care in Arizona managed Medicaid compared with FFS in New Mexico. Maternity care and pregnancy outcomes were similar in the 2 states.

Less well studied is the possible effect of managed Medicaid on chronically ill populations. The review of nursing home records in Arizona's managed Medicaid and Medicaid FFS plans in New Mexico suggested that Arizona's elderly and disabled were more likely to have pressure sores, fever, or a catheter inserted and were less likely to be offered an influenza vaccine. ${ }^{4}$ The fall and fracture rates and psychotropic drug use were similar between the 2 states.

Assessment of care quality is particularly important for children with chronic conditions because of higher ongoing costs associated with managing their illness. ${ }^{5-7}$ There is limited research examining quality of care received by Medicaid children with chronic conditions in managed Medicaid plans compared with an FFS plan..$^{8-10}$ One study reported the Health Employer Data and Information Set (HEDIS) asthma measure for comparing quality of care received by New York Medicaid children enrolled in FFS with managed Medicaid. ${ }^{8}$ Using administrative datasets, the study compared the percentage of children with asthma aged 5 to 17 in the 2 plans. These children were continuously enrolled in their health plans for 2 years and received at least 1 medication acceptable as primary therapy for long-term control. Those in managed Medicaid had a higher rate on the quality measure compared with those in FFS (53\% vs. 51\%, $P<0.001)$.

Shields et al. (2002) compared the process of care received by Massachusetts Medicaid children with asthma served by 2 managed Medicaid plans-a primary care plan and a staff model health maintenance organization (HMO) -with an FFS plan. ${ }^{9}$ The primary care plan comprised provider groups such as community health centers, hospital outpatient departments, and solo/group physicians. Adjusted analyses compared solo/ group physicians in primary care plans with other provider types, including 2 primary care plans (community health centers and hospital outpatient departments), an HMO, and an FFS plan. The comparison was conducted on 5 process-ofcare measures derived from administrative claims, including (1) access to a specialist for children with persistent asthma and for children with moderate/severe asthma, (2) appropriate use of controller medications, (3) overuse of beta ${ }_{2}$-agonists, and $(4,5)$ follow-up visits after asthma-related emergency department visits or hospitalization. Compared with solo/group physician plans, the FFS plan performed similarly on 4 measures and better on 1 measure (follow-up visits after asthma-related hospitalization).

Comparison of health care use and medication use patterns for children with asthma covered under Medicaid or nonMedicaid (commercial) payers within the same HMO found higher use of emergency department visits and hospitalizations and lower use of controller medications in Medicaid children compared with non-Medicaid children. ${ }^{10}$ Ambulatory visits and use of beta $_{2}$-agonists was similar in the 2 populations. This study by Finkelstein et al. examined the quality of care for Medicaid children compared with non-Medicaid children enrolled in the same health plan but did not report on quality of care for Medicaid members in managed Medicaid plans compared with FFS plans.

We conducted the present study as part of the oversight of the implementation of the Maryland HealthChoice program, a mandatory managed Medicaid program that enrolls most Medicaid recipients in the state. Previous evaluations of this program used cross-sectional designs to focus on access issues such as ambulatory care, well-child visits, and emergency department visits for the general population and, to a limited extent, for children with selected chronic conditions. ${ }^{11,12}$ The state's professional review organization (PRO), the Delmarva Foundation, also examined quality of care for children with 


\section{Quality of Drug Treatment of Childhood Persistent Asthma in Maryland Medicaid \\ Recipients in Transition From Managed Fee for Service to Managed Capitation}

chronic conditions under the HealthChoice program through onsite audits. ${ }^{13}$ To our knowledge, no published study has used a longitudinal design to compare the HealthChoice capitated managed Medicaid plan to pre-HealthChoice managed FFS.

To fill this gap, we analyzed the experiences of children with asthma in HealthChoice. We chose asthma because of its high prevalence among Maryland Medicaid children and because complications associated with the disease can be prevented by proper treatment and monitoring, outcomes that are anticipated in managed care. Our objective was to determine whether quality of prescribing for pediatric asthma treatment improved during the transition from FFS to the HealthChoice program. We hypothesized that HealthChoice managed Medicaid plans would be better able to communicate with providers and emphasize standards of care according to the national asthma guidelines than would an FFS plan. ${ }^{14}$ HealthChoice plans would do this by relying on better monitoring of drug therapy to minimize the costs of hospitalization and emergency department treatments. To test our hypothesis, we retrospectively followed a cohort of children with asthma from FFS into HealthChoice and tracked the quality of their asthma treatment using a variant of the HEDIS measure of quality of asthma treatment. This variant was the proportion of study subjects receiving 2 or more claims for short-acting beta ${ }_{2}$-agonists (SABAs: metered-dose inhalers, nebulizers, or oral forms) as rescue agents in a 6-month period (a marker for persistent asthma) who also received at least 1 controller medication (an inhaled corticosteroid, a mast-cell stabilizer, and/or a leukotriene-receptor modifier) concurrently.

\section{- Maryland's HealthChoice Managed Medicaid Program}

Authorized under a federal section 1115 waiver, Maryland implemented its full-risk HealthChoice managed Medicaid program in July 1997 and required mandatory enrollment of most of the state's Medicaid recipients in 1 of 9 managed care plans (by 2002, the number of individual plans declined to 6 because of withdrawals and mergers). Excluded from the mandatory HealthChoice program were certain recipients, such as those dually eligible for both Medicaid and Medicare, and residents of nursing homes, chronic hospitals, and mental hospitals. ${ }^{11,12}$ In addition, individuals with qualifying "rare and expensive" medical conditions were permitted to opt out into an FFS case management plan. Eligible beneficiaries received a unified provider directory serving their region and listing HealthChoice MCOs. Beneficiaries were given 21 days to select both a health plan and provider (children in foster care were given 60 days) after which they were automatically assigned to a managed Medicaid plan based on geographic location and other factors. Five guiding values defined the purpose of HealthChoice: (1) provide a patient-focused system with a medical home for all patients; (2) provide comprehensive, prevention-oriented systems of care; (3) build on the strengths of the Maryland health care system; (4) achieve better value and predictability for state expenditures; and (5) hold MCOs accountable for high quality of care. ${ }^{11,12}$

Under the program, the plans were contracted to provide the full range of health care services covered under the HealthChoice MCO benefit package, which was equivalent to benefits covered by the Medicaid FFS plan as of January 1, 1997 (except for certain carved-out services). The payment for enrollees' health care services was based on a fixed monthly capitation rate, risk adjusted by the state for the enrollees' health status.

The implementation of the HealthChoice program replaced other forms of less comprehensive managed Medicaid programs in the state, including an $\mathrm{HMO}$ program and a primary care case management program called Maryland's Access to Care (MAC). ${ }^{11,12,15}$ The HMO program had been available since 1975 and had provided Medicaid services to recipients who enrolled voluntarily. The enrollment in the HMO program was limited; just about $20 \%$ of Medicaid recipients were enrolled in an HMO in 1997 before HealthChoice. ${ }^{11}$

The MAC program had been a mandatory managed FFS Medicaid program, in operation since 1991. Under this program, most Medicaid recipients, except those enrolled in an HMO and some others meeting certain criteria, were required to either choose or be assigned to a primary medical provider (PMP). ${ }^{15}$ The PMPs acted as gatekeepers to the health care system, providing primary and preventive care and making referrals to specialty care as needed. The PMPs were paid on an FFS basis for all services provided and were not put at direct financial risk for the patients enrolled with them.

The MAC program, however, lacked the infrastructure to implement a comprehensive care management process (such as disease management and utilization review) for its enrollees and lacked incentives to limit the volume of services. ${ }^{11}$ In the end, despite improving access to primary and preventive care, the program cost more to the state than the program it replaced. ${ }^{15}$ With a fixed capitation rate arrangement under HealthChoice, the state placed health plans at direct financial risk and provided them with incentives to implement appropriate care management processes to control costs and improve access and quality.

Maryland's HealthChoice managed Medicaid program enrolled more than 300,000 recipients between July 1997 and January 1998. The state required all plans to submit detailed patient-provider encounter data (also known as "pseudo" claims, since they were used to track use of services and not to give direct payment to providers) in order to set risk-adjusted capitation rates and to monitor quality. The state contracted with the Delmarva Foundation to provide quality oversight through periodic audits and client satisfaction surveys.

\section{Methods}

Our 4-year study of the HealthChoice program involving 


\section{Quality of Drug Treatment of Childhood Persistent Asthma in Maryland Medicaid \\ Recipients in Transition From Managed Fee for Service to Managed Capitation}

children with asthma, dubbed Project INHALE (INitiative to Help Asthmatics Live Easier), is described elsewhere. ${ }^{16,17}$ Project INHALE was designed to use FFS claims, plan enrollment data, and MCO encounter forms to follow members of the study cohort through the transition into HealthChoice. Study subjects ( $\mathrm{n}=5,804)$ were selected on the basis of a review of Medicaid enrollment records and medical and pharmacy FFS claims filed between June 1, 1996, and December 31, 1997. Selection criteria included age (between 5 and 18 years at the time of MCO enrollment), a minimum of 3 months of continuous Medicaid enrollment during the baseline FFS period, and evidence of asthma at baseline. Evidence of asthma was based on meeting 1 or more of the following 3 criteria during the baseline period of at least 3 months: (1) at least 1 medical or hospitalization claim with an asthma diagnosis (International Classification of Diseases, Ninth Revisions Clinical Modification [ICD-9-CM] code 493.x) and at least 1 claim for an asthma-related drug, (2) at least 2 medical or hospitalization claims with an asthma diagnosis, or (3) at least 3 pharmacy claims for an asthma-related drug. Children with a diagnosis of cystic fibrosis (ICD-9-CM code 277.0x) were excluded from the study. The study was approved by institutional review boards at the University of Maryland at Baltimore and the Maryland Department of Health and Mental Hygiene.

We limited the present study to enrollees in the 4 largest HealthChoice managed Medicaid plans $(n=3,721)$. Using unique recipient identifiers, we merged FFS claims, plan enrollment data, and managed Medicaid encounter forms and created data files with information on Medicaid eligibility, setting (FFS or managed Medicaid with plan indicators), dates of managed Medicaid enrollment and disenrollment, and prescription drug use before and after transition to managed Medicaid. Date of first managed Medicaid enrollment was defined as the index date of the study, and enrollment plan was defined as the index plan. We followed each plan member in the study from June 1, 1996 (or, if later, first Medicaid eligibility date), through December 31, 2000 (or, if earlier, last Medicaid eligibility date). We censored observations after an enrollee lost Medicaid eligibility or switched from the index plan.

To measure the quality of asthma treatment during the FFS and managed Medicaid periods, we used a variant of the National Committee for Quality Assurance's HEDIS measure for the use of appropriate medications for people with persistent asthma. The HEDIS asthma measure identifies individuals with persistent asthma based on their medical and/or asthma drug use in one year, and then in the next year, determines the proportion of these individuals who used at least 1 medication recommended by the National Heart, Lung, and Blood Institute as primary therapy for long-term control of asthma. ${ }^{18}$ These medications, referred to as controller medications, include inhaled corticosteroids, mastcell stabilizers, and leukotriene-receptor modifiers.

In Maryland, the managed Medicaid plans had inconsistent and erratic reporting (i.e., variations across plans and time) of encounter data, causing the HEDIS asthma measure to potentially produce invalid or misleading results if applied in its original form. ${ }^{19}$ The inconsistent and erratic encounter data reporting may have occurred for several reasons, including (1) lack of incentives for plans to submit data since there was no direct tie with reimbursement, and (2) data collection problems at both ends; that is, by plans from their network providers or subcontractors and by states from individual plans because of strict data submission requirements and standards embedded in the electronic systems. ${ }^{19}$ The latter is especially true for pharmacy services that are generally outsourced to a third-party administrator, which must collect data from network providers in a format compatible with the MCOs' systems, which, in turn, had to conform with the state's system. Eventually, these data problems were solved through active efforts on the part of both the state and MCOs, but in the interim, the problem with data completeness remained. ${ }^{11}$

We adapted the HEDIS asthma measure to our current situation. To be consistent with the literature, we defined individuals with persistent asthma based on their receipt of 2 or more pharmacy claims for rescue medications (SABAs-metereddose inhalers, nebulizers, or oral forms) in a 6-month period. ${ }^{16}$ Once we identified patients in this manner as having persistent asthma in a 6-month period, we determined the number of these patients who also had at least 1 pharmacy claim for a controller medication in the same 6-month period. Presence of a pharmacy claim for a rescue or a controller medication was operationally defined as the receipt of the medication. Thus, we defined the quality indicator measure as the proportion of study subjects who had 2 or more pharmacy claims for beta 2 -agonists, and who had at least a pharmacy claim for a controller medication during the same 6-month period. Higher values on this measure indicate better quality of asthma care. The quality indicator measure for a 6-month period was computed as follows:

\# of subjects who had at least 1 pharmacy claim

for a controller medication among those who had

2 or more pharmacy claims for beta ${ }_{2}$-agonists

\# of subjects who had 2 or more

pharmacy claims for beta $_{2}$-agonists

We expected our quality indicator measure to overcome the incomplete administrative claims reporting by the HealthChoice managed Medicaid plans, since the measure was based on a proportion of 2 types of asthma medications (controller and rescue) and there was no a priori reason to believe that prescription records for 1 type were more likely to be reported than for the other type; that is, administrative claims for a given patient would be either present or absent during any given 6 -month period. We formally tested this presumption in an 


\section{FIGURE 1 Schematic Diagram of Study Design With 2 Examples of Patient Enrollment Over Eight 6-Month Time Periods of Study}
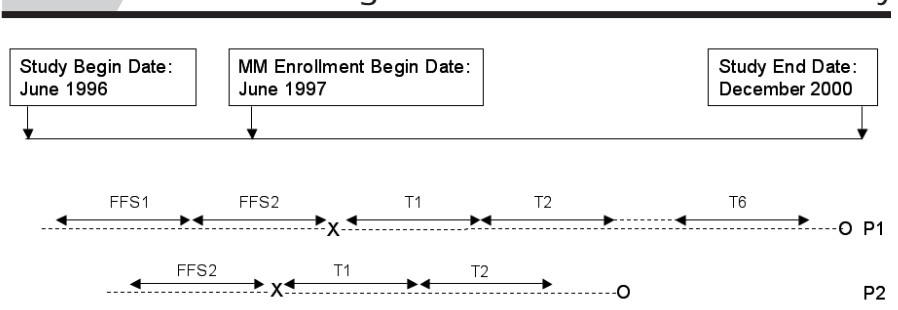

FFF1 \& FFS2 =6-month periods in baseline managed-fee for service.

$P 1=$ Person 1 enrolled in an index study managed Medicaid plan.

P2 = Person 2 enrolled in an index study managed Medicaid plan.

T1 through T6 $=6$-month periods in managed Medicaid plan.

$X=$ index date of plan enrollment.

$O=$ loss of Medicaid eligibility or switch to an MM Care plan other than the index plan. MM= managed Medicaid.

\section{FIGURE 2 Study Sample Selection}

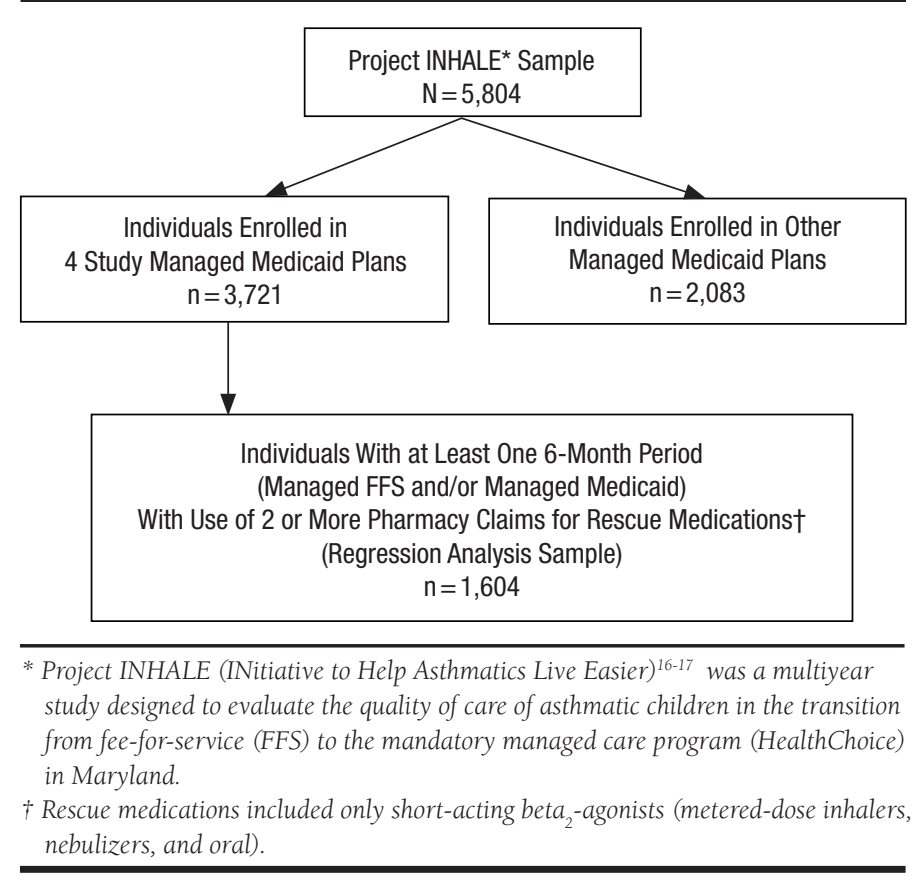

earlier study and found the quality indicator measure to be robust to data loss. ${ }^{17}$

We characterized the study sample on key sociodemographic factors, including age, sex, and race. As noted, the analyses focused on individuals with 2 or more rescue medication prescriptions in a 6-month period. For each person in the cohort, 6-month periods were defined based on time before and after the person's index date of enrollment in a managed Medicaid plan. Two 6-month periods, FFS1 and FFS2, were defined as the FFS baseline, and six 6-month periods, $\mathrm{Tl}$ to T6, were defined as the managed Medicaid exposure (Figure 1). For each 6-month period, we identified all individuals continuously enrolled during the 6-month period who had 2 or more pharmacy claims for rescue medications (the denominator of the quality indicator). Then we determined the number of these patients who also had at least 1 pharmacy claim for controller medication (the numerator of the quality indicator). Using these data, we calculated mean quality indicator rates for each 6-month period before and after HealthChoice. To assess the trends in the quality indicator rates over time, we plotted quality indicator rates from FFS1 through T6.

To formally assess the statistical significance of observed trends, we fit log-binomial generalized estimating equations (GEE) regression models, which accounted for the correlation between repeated measures from the same individuals over time. ${ }^{20,21}$ The unit of analysis in these models was each 6-month period for each person. Only those periods were used in which the patient had at least 2 pharmacy claims for SABA (rescue) medication.

The outcome variable was binary: whether the patient received a controller medication. The exposure (independent) variable was whether it was an FFS or managed Medicaid period. In these models, we controlled for demographics (age, sex, and race) and season. The analysis accounted for increasing age of individuals over time. Season was dichotomized as fall and spring and defined on the basis of the most days falling between June and November (fall season) and December and May (spring season). For these regression analyses, we collapsed the baseline periods (FFS1 and FFS2) into a single reference category. Thus, the final pooled dataset consisted of individuals with only FFS, only managed Medicaid, or both FFS and managed Medicaid observations.

Individuals with only FFS (or only managed Medicaid) were those who either did not have a 6-month continuous eligibility during the managed Medicaid periods (or FFS periods) or did not have 2 or more prescriptions of rescue medications in the 6-month period if they were continuously eligible during the managed Medicaid periods (or FFS periods). The GEE analyses sample restricted to individuals with at least 1 eligible 6-month period in FFS or managed Medicaid resulted in a total of 1,604 unique people contributing 3,114 person-observations over 6 months. Figure 2 describes the study sample selection up to the sample for regression analysis. Table 1 describes the study measures employed in the regression analyses.

We used 2 models for the GEE analyses. Model 1 estimated whether managed Medicaid made a difference in the receipt of a controller medication. The model had the following structure: 
$\log (\mathrm{p})=\alpha+\beta_{1}$ managed Medicaid

$+\beta_{2}$ Demographics $+\beta_{3}$ Season

where $\log (\mathrm{p})$ is the $\log$ of the probability that the individual with 2 or more rescue medications received concurrent controller medication during the 6-month observation period; managed Medicaid is a binary indicator of whether the 6-month period was in managed Medicaid; Demographics is a vector of predictors (age, sex, race), and Season is an indicator of whether the 6-month period fell in the fall. In this model, $\beta_{1}$ coefficient represents the relative probability of receiving a controller medication in a managed Medicaid plan compared with the FFS baseline. The coefficient is interpretable as a percentage increase or decrease in the probability of receiving a controller medication during managed Medicaid compared with the baseline.

Model 2 estimated the change in the relative probability of receiving a controller medication over time during managed Medicaid periods. For estimating this model, we added terms interacting the main managed Medicaid impact with a time variable coded 1 to 6 , representing the observation periods after baseline. The model structure was as follows:

$\log (p)=\alpha+\beta_{1}$ managed Medicaid $+\beta_{2}$ managed Medicaid*Time $+\beta_{3}$ Demographics $+\beta_{4}$ Season

In this model, the parameter associated with the interaction term $\left(\beta_{2}\right)$ was interpretable as the degree to which the relative probability of using a controller changed over time within managed Medicaid relative to FFS. We conducted all analyses using SAS v. 8.2 (SAS Institute, Inc., Cary, NC).

\section{Results}

The mean age of the study sample was 10.5 (standard deviation, 3.8) years, and males constituted $56.8 \%$ of the sample. African Americans represented $59.1 \%$, whites $36.4 \%$, and others $3.5 \%$ of the sample. The plan enrollees were mostly similar in sociodemographic characteristics across 4 plans included in the study (data not shown).

The raw data to compute quality indicator rates and the computed rates from the 2 baseline FFS periods (FFS1 and FFS2) and 6 follow-up periods of managed Medicaid exposure (T1 to T6) are shown in Table 2. The quality indicator rates are presented in graphical form in Figure 3. The quality indicator rates for the 2 FFS baseline periods were $62 \%$ during FFS1 and 57\% during FFS2 (Table 2). During the 6 managed Medicaid periods, the quality indicator values ranged from a low value of $56 \%$ during $\mathrm{T} 1$ to a high value of $66 \%$ during T5.

The multivariate results from the 2 GEE models are presented in Table 3. The first panel shows the relative risk (RR) of receiving controller medications during the managed Medicaid plan periods compared with the FFS baseline periods (Model 1). Overall, the relative probability of receiving a controller
(TABLE 1$)$ Study Measures for the Regression Analysis

\begin{tabular}{l|l}
\hline Variable & Variable Specification \\
\hline \begin{tabular}{l} 
Dependent variable \\
\hline $\begin{array}{l}\text { Receipt of at least l pharmacy claim for a } \\
\text { controller medication }\end{array}$
\end{tabular} \\
\hline \begin{tabular}{l} 
Primary independent variables \\
\hline Managed Medicaid
\end{tabular} \\
\hline Managed Medicaid x time & Yes/No \\
\hline Explanatory variables & Continuous \\
\hline Age & Yes/No \\
\hline Gender & Yes/No \\
\hline White/non-white race & Yes/No \\
\hline Fall/spring season &
\end{tabular}

TABLE 2 Quality Indicator Rates for 2 Time Periods in Managed-Fee for Service (FFS) and 6 Time Periods in Managed-Medicaid for the Study Cohorts

\begin{tabular}{|c|c|c|c|}
\hline & $\begin{array}{l}\text { No. With } \\
2 \text { or More } \\
\text { Prescriptions } \\
\text { for Rescue* } \\
\text { Medications }\end{array}$ & $\begin{array}{l}\text { No. With } \\
1 \text { or More } \\
\text { Prescriptions } \\
\text { for Controller } \dagger \\
\text { Medication } \\
\end{array}$ & $\begin{array}{c}\text { Quality } \\
\text { Indicator Rate } \\
(95 \% \mathrm{CI})\end{array}$ \\
\hline FFS1 time period & 735 & 459 & $0.62(0.59-0.66)$ \\
\hline FFS2 time period & 837 & 474 & $0.57(0.53-0.60)$ \\
\hline Tl time period & 223 & 124 & $0.56(0.49-0.62)$ \\
\hline T2 time period & 186 & 105 & $0.57(0.49-0.63)$ \\
\hline T3 time period & 300 & 178 & $0.59(0.54-0.65)$ \\
\hline T4 time period & 256 & 155 & $0.61(0.54-0.66)$ \\
\hline T5 time period & 287 & 190 & $0.66(0.61-0.71)$ \\
\hline T6 time period & 290 & 170 & $0.59(0.53-0.64)$ \\
\hline \multicolumn{4}{|c|}{$\begin{array}{l}\text { * Rescue medications included only short-acting beta }{ }_{2} \text {-agonists (metered-dose } \\
\text { inhalers, nebulizers, and oral dose forms). } \\
+ \text { Controller medications included inhaled corticosteroids, mast-cell stabilizers, and } \\
\text { leukotriene-receptor modifiers. } \\
\text { CI=confidence interval. }\end{array}$} \\
\hline
\end{tabular}

medication appeared to be slightly greater (1\%) during managed Medicaid than during FFS, but the effect was not statistically significant (RR, 1.01; 95\% confidence interval [CI], 0.95-1.08).

The second panel in Table 3 shows the results for the changes in the relative probability of receiving controller medications over time during the managed Medicaid periods (Model 2). Overall, there was no trend in the quality indicator rates over time during the managed Medicaid periods compared with the FFS baseline (RR, 1.02; 95\% CI, 0.99-1.04). 
FIGURE 3 Proportion of Patients With at Least 1 Pharmacy Claim for Controller* Medication Among Those With at Least 2 Pharmacy Claims for Rescue* Medications During the Fee-For-Service and Managed-Medicaid 6-Month Periods†

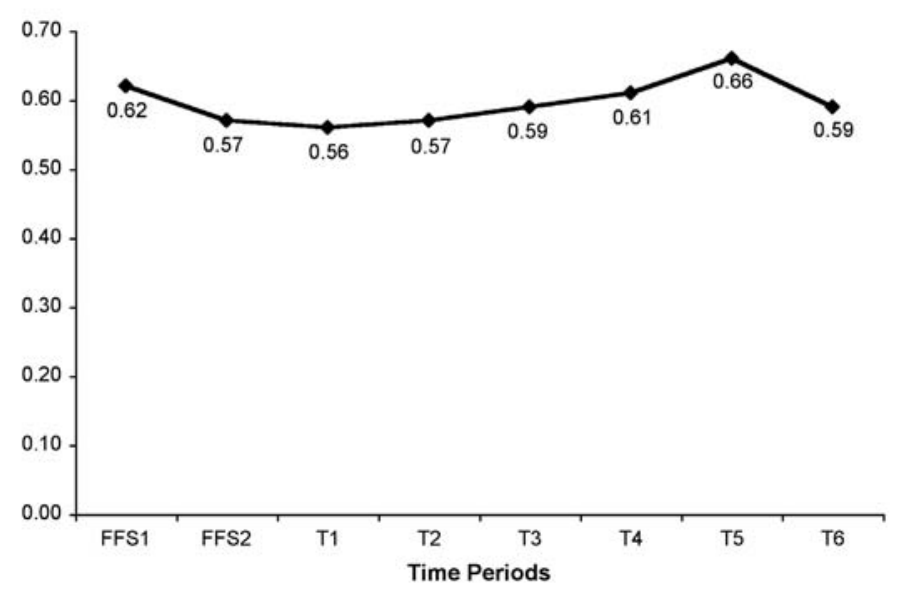

* Controller medications included inhaled corticosteroids, mast-cell stabilizers, and leukotriene-receptor modifiers. Rescue medications included only short-acting beta ${ }_{2}$-agonists (metered-dose inhalers, nebulizers, and oral dose forms).

+ FFS1 and FFS2 were baseline managed-fee-for-service periods and T1 to T6 were managed Medicaid periods

(TABLE 3 Impact of Transition to ManagedMedicaid on the Relative Risk of Receiving at Least 1 Controller Medication* Among Those Receiving 2 or More Rescue Medications in the Same 6-Month Period

\begin{tabular}{l|c|c}
\hline Model & Relative Risk & $95 \%$ CI \\
\hline Model 1 & 1.01 & $0.95-1.08$ \\
Managed Medicaid & 0.93 & \\
\hline Model 2 & 1.02 & $0.83-1.05$ \\
Managed Medicaid & $0.99-1.04$ \\
\hline
\end{tabular}

* Controller medications included inhaled corticosteroids, mast-cell stabilizers, and leukotriene-receptor modifiers.

+ Rescue medications included only short-acting beta ${ }_{2}$-agonists (metered-dose inhalers, nebulizers, and oral dose forms).

Note: The time variable ranged from 0 to 6, representing fee-for-service baseline 6-month periods (time $=0$ ) and managed Medicaid plan follow-up 6-month periods (time $=1$ to 6)

Reference group: fee for service.

Control variables included age, gender, race, and season and their coefficients for Model 1 were age: $0.99(0.98,1.00)$, gender: $1.00(0.92,1.08)$, race: $1.06(0.98$, 1.15), and fall season: $0.98(0.93,1.03) . P<0.05$.

$\mathrm{CI}=$ confidence interval.

\section{Discussion}

The 1990s witnessed a major shift of Medicaid enrollees from FFS into managed Medicaid plans. Although the new health care delivery system promised to improve access and quality of care while keeping costs down, it is important to evaluate if these goals were actually achieved. These evaluations of program outcomes may attract more attention for certain subpopulations, such as children with chronic illnesses like asthma. Quality indicators based on national treatment guidelines may provide a useful tool in this regard.

The present study evaluated one small aspect of quality of care for children with asthma enrolled in the Maryland HealthChoice program. Since we were forewarned about missing encounter data during managed Medicaid periods, ${ }^{19}$ we used a HEDISrelated quality indicator that we found in an earlier study to be robust to data loss. ${ }^{17}$ Briefly, in that study, in a series of Monte Carlo simulations, we artificially simulated several degrees (5\% to $95 \%$ ) and types of data loss (systematic, i.e., missing data for entire periods, and random, i.e., missing data across periods) in the FFS portion of our dataset. We measured the percentage of deterioration in the quality indicator we observed on the basis of the complete data, which gave an indication of the robustness of the measure to data loss (i.e., low deterioration in quality indicator indicated high robustness to data loss). Although the measure was not validated against medical charts, it was found to be robust to data loss in simulation experiments to the extent that the data loss did not exceed 35\%, meaning that the measure was found to be insensitive to data loss of the magnitude up to $35 \%$. The state's own analysis of various types of encounter data showed pharmacy data to be more than $85 \%$ complete across MCOs during the study period (relatively less-complete data elements were physician: $75 \%$ to $90 \%$ and inpatient hospital: $50 \%$ to $75 \%$ ), reassuring the use of the asthma quality measure for producing valid results in the present study. ${ }^{19}$

To evaluate how Maryland's HealthChoice program affected the quality of care, we retrospectively tracked asthmatic children enrolled into the 4 largest managed Medicaid plans during their transition from FFS to HealthChoice. Specifically, we examined whether there was any significant improvement or deterioration in the quality of drug use for the treatment of persistent asthma as measured by the quality indicator during the transition. We found none. This finding of no change from FFS to managed Medicaid compares with improved access to care found in a separate study of the HealthChoice program. In that study, over the approximate time period of our analysis, Medicaid children, on average, had a 3\% higher rate of ambulatory care visits during the HealthChoice periods than during FFS periods. ${ }^{12}$ However, improved access to care seen in that study was not reflected in our study in improved quality of care.

In another study of the quality of pediatric asthma care in Maryland Medicaid, the state's PRO, the Delmarva Foundation, found that a larger proportion of chart reviews conducted at 


\section{Quality of Drug Treatment of Childhood Persistent Asthma in Maryland Medicaid \\ Recipients in Transition From Managed Fee for Service to Managed Capitation}

managed Medicaid plan sites, 84\% in 2000 compared with 55\% in 1998, met the state's specified quality standards for pediatric asthma care. ${ }^{13}$ This study performed by the PRO covered the same time period as the present study, but the method for quality assessment differed from our study (i.e., chart review versus analysis of administrative claims), and there was no comparison of outcomes in managed Medicaid versus FFS.

Our findings are consistent with another evaluation of Maryland HealthChoice for substance abuse treatment patterns and outcomes. ${ }^{22}$ In that study, Ettner et al (2003) linked Medicaid eligibility files with treatment provider records and found that treatment utilization and outcomes did not differ in those entering the treatment in MCOs than in FFS. And although utilization was higher and outcomes were better for a subgroup of beneficiaries transitioning from FFS to MCO during the study period, the positive effect was explained to be most likely due to the switching of providers and reevaluations. ${ }^{22}$

Our findings are also consistent with a study by Shields et al. (2002), who reported no difference in quality for Medicaid childhood asthma treatment on the basis of a measure similar to the one used in the present study. ${ }^{9}$ The quality measure used by Shields et al. was the proportion of patients who received at least 1 controller medication among patients who received 3 or more months' supply of beta $2_{2}$-agonists (aerosols, nebulizer, or syrup) within a 6-month period. Shields et al. found that compared with children in a managed Medicaid group, children enrollees in FFS were equally likely to receive the controller medication (odds ratio, 1.17; 95\% CI, 0.82-1.67).

Roohan et al., in a study reported more recently (2006), compared asthma quality of care in managed Medicaid with Medicaid FFS in New York State and found better quality under managed Medicaid: the proportion of moderate to severe asthmatics who received a controller medication was 53\% in managed Medicaid compared with 51\% in FFS. ${ }^{8}$ Our study did not find improved quality in managed Medicaid, but the findings of Roohan et al., while statistically significant, represent a small absolute difference in the comparison of managed Medicaid with FFS Medicaid.

There are at least 3 possible explanations for the null findings in the present study. One, it may be that nothing happened; that provider practice patterns are consistent and not sensitive to health plan organizational structure. An analysis of whether there was some change in the makeup of health care providers for these children with asthma in the managed Medicaid transition would have been helpful in shedding some light on this issue; however, physician-level information was not available to us.

Two, our FFS care was delivered under the MAC program, and the care delivery was already at a relatively higher quality level before HealthChoice. Previous evaluations of the MAC program have reported improved access and higher quality of care under MAC than under traditional FFS. Schoenman et al. found that, compared with traditional FFS, the MAC program increased the use of primary and preventive care services and of prescription drugs. ${ }^{15}$ Similarly, Gadomski et al. (1998) found that the MAC program increased the probability of preventive and any ambulatory visits and reduced the probability of avoidable and any hospitalizations when compared with traditional FFS. ${ }^{23}$ It is possible that the quality of care in certain areas, such as asthma care, reached ceiling effects before HealthChoice via MAC's primary case management program; however, it is not possible to test this possibility empirically.

Three, we did not have sufficient power to detect significant differences due to the sample size limitations.

\section{Limitations}

The first and foremost limitation in the present study is the threat to validity caused by missing administrative data, particularly in the managed Medicaid time periods in which the administrative data were not the basis for provider reimbursement. While calculation of our quality indicator was previously found to be robust to missing data, the sample sizes in the present study might not be large enough to show statistical significance when the change in the quality indicator appeared to be clinically meaningful. We did not validate the observed quality indicator rates against the medical records, which would presumably rule out any potential bias due to missing encounter data.

Second, as in any longitudinal study without a control group, the dependent measure can be influenced by any number of unobservable factors, such as change in patient or provider behavior over time. New asthma guidelines were released before the transition of the majority of our sample into managed care. ${ }^{14}$ However, we do not believe this affected our results, since the 1997 guidelines did not differ from previously published 1991 guidelines in the particular aspect that we evaluated in our study; namely, the treatment of persistent asthma. ${ }^{24}$

Third, we compared quality of asthma care in managed Medicaid with prior enrollment in managed FFS on the basis of only 1 measure. Using other quality measures, including access to care, provider follow-up visits, or receipt of controller drugs after an emergency department visit or hospital discharge, with an asthma diagnosis might have produced different results.

Fourth, the present study was not able to examine differences in care delivered by individual health plans because of the sample size limitations.

\section{Conclusion}

Over the entire 4-year period of this study, approximately $56 \%$ to $66 \%$ of Medicaid children in any given 6-month period received a controller medication in addition to at least 2 pharmacy claims for a SABA. There was no difference in the quality of asthma care between capitated managed Medicaid plans and Medicaid managed FFS plans for these children with persistent asthma. 


\section{Quality of Drug Treatment of Childhood Persistent Asthma in Maryland Medicaid Recipients in Transition From Managed Fee for Service to Managed Capitation}

\section{Authors}

PUNEET K. SINGHAL, BPharm, PhD, is manager, Outcomes Research, Merck $\mathcal{E}$ Co., Inc., West Point, Pennsylvania (at the time of the study he was a graduate research assistant, University of Maryland at Baltimore School of Pharmacy); ILENE ZUCKERMAN, PharmD, PhD, and LAURENCE MAGDER, PhD, is an associate professor, University of Maryland School of Medicine; BRUCE STUART, PhD, is a professor and director, Peter Lamy Center for Drug Therapy and Aging, University of Maryland at Baltimore School of Pharmacy; HAYA RUBIN, MD, PhD, is executive director, Palo Alto Medical Foundation Research Institute, Palo Alto, California.

AUTHOR CORRESPONDENCE: Puneet K. Singhal, BPharm, PhD, Manager, Outcomes Research, Merck \& Co., Inc., 770 Sumneytown Pike, WP97-A241, West Point, PA 19486. Tel: (215) 652-1538 (office); (215) 368-1265 (home); Fax: (215) 993-3082; E-mail:puneet_singhal@merck.com

\section{ACKNOWLEDGMENT}

The authors would like to thank Dr. Cheryl Fahlman, health researcher, Mathematica Policy Research, Washington, DC, for assistance with the literature review in this study and the editors and reviewers for their assistance with this article.

\section{DISCLOSURE}

Funding for this research was provided by a grant from the Agency for Healthcare Research and Quality to the University of Maryland, which was obtained by author Bruce Stuart. Author Puneet K. Singhal is employed by Merck. None of the authors disclose potential bias or conflict of interest relating to this article. The results of this study were presented at the 19th Academy Health Annual Research Meeting in June 2004 in San Diego, California.

Singhal served as principal author of the study. Study concept and design were contributed primarily by Stuart, with a significant contribution from authors Laurence Magder and Ilene Zuckerman and additional input from author Haya Rubin. Data collection, data interpretation, and writing and revision of the manuscript were the work of all authors.

\section{REFERENCES}

1. Centers for Medicare $\&$ Medicaid Services, U.S. Dept. of Health and Human Services. Medicaid Managed Care Enrollment Report as of June 30, 2005.

Available at: http://www.cms.hhs.gov/MedicaidDataSourcesGenInfo/ Downloads/mmcer05.pdf. Accessed March 31, 2005.

2. Medicaid: States turn to managed care to improve access and control costs. Washington, D.C.: U.S. General Accounting Office; March 1993. Report no.: GAO/HRD-93-46.

3. Freund D, Rossiter LF, Fox PD, et al. Evaluation of the Medicaid competition demonstrations. Health Care Financ Rev. 1989;11(2):81-97.

4. McCall N. Lessons from Arizona's Medicaid managed care program. Health Aff (Millwood). 1997;16(4):194-99.

5. Neff JM, Anderson G. Protecting children with chronic illness in a competitive marketplace. JAMA. 1995;274:1866-69.

6. Newacheck PW, Stein RE, Walker DK, et al. Monitoring and evaluating managed care for children with chronic illnesses and disabilities. Pediatrics. 1995;98:952-58.
7. Kuhlthau K, Walker DK, Perrin JM, et al. Assessing managed care for children with chronic conditions. Health Aff. (Millwood). 1998;17:42-52.

8. Roohan PJ, Butch JM, Anarella JP, et al. Quality measurement in Medicaid managed care and fee-for-service. Am J Med Qual. 2006;21:185-91.

9. Shields AE, Finkelstein JA, Comstock C, et al. Process of care for Medicaidenrolled children with asthma: served by community health centers and other providers. Med Care. 2002;40:303-14.

10. Finkelstein JA, Barton MB, Donahue JG, et al. Comparing asthma care for Medicaid and non-Medicaid children in a health maintenance organization. Arch Pediatr Adolesc Med. 2000;154:563-68.

11. Maryland Department of Health and Mental Hygiene. HealthChoice evaluation: final report $\&$ recommendations: January 15, 2002. Available at: http://www.dhmh.state.md.us/mma/healthchoice/hcevalprestabcon.html. Accessed December 27, 2004.

12. Chang DI, Burton A, O'Brien J, et al. Honesty as good policy: evaluating Maryland's Medicaid managed care program. Milbank Q. 2003;81:389-414.

13. HealthChoice program CY2000 annual quality of care audit fact sheet. Baltimore, MD: Maryland Department of Health and Mental Hygiene; 2001.

14. Guidelines for the diagnosis and management of asthma. Bethesda, MD U.S. Dept. of Health and Human Services, National Heart, Lung, and Blood Institute, National Asthma Education and Prevention Program; 1997. Expert panel report 2. Publication 97-4051.

15. Schoenman JA, Evans WN, Schur CL. Primary care case management for Medicaid recipients: evaluation of the Maryland access to care program. Inquiry. 1997;34:155-70

16. Zuckerman IH, Stuart B, Magder LS, et al. Adherence of asthma treatment guidelines among children in the Maryland Medicaid program. Curr Ther Res Clin Exp. 2000;61:912-24

17. Stuart B, Singhal PK, Zuckerman IH, et al. How robust are health plan quality indicators to data loss? A Monte Carlo simulation study of pediatric asthma treatment. Health Serv Res. 2003;38:1547-61

18. Glauber JH. Does the HEDIS asthma measure go far enough? Am J Manag Care. 2001;7(6):575-79.

19. HealthChoice encounter data collection: challenges, accomplishments, and plan performance 1999-2000. Baltimore, MD: Maryland Department of Health and Mental Hygiene; 2000.

20. Zeger SL, Liang KY. Longitudinal data analysis for discrete and continuous outcomes. Biometrics. 1986;42:121-30.

21. McNutt LA, Wu C, Xue X, et al. Estimating the relative risk in cohort studies and clinical trials of common outcomes. Am J Epidemiol. 2003;157:940-43.

22. Ettner SL, Denmean G, Dilonardo J, Cao H, Belanger AJ. The impact of managed care on the substance abuse treatment patterns and outcomes of Medicaid beneficiaries: Maryland's HealthChoice program. J Beh Health Ser Res. 2003;30(10):41-62.

23. Gadomski A, Jenkins P, Nicholas M. Impact of a Medicaid primary care provider and preventive care on pediatric hospitalization. Pediatrics. 1998;101(3):E1.

24. Guidelines for the diagnosis and management of asthma. Bethesda, MD U.S. Dept. of Health and Human Services, National Heart, Lung, and Blood Institute, National Asthma Education and Prevention Program; 1991. Expert panel report. Publication 91-3642. 